\title{
Reinternamentos Hospitalares num Serviço de Pedopsiquiatria: Taxa de Readmissão e Fatores de Risco
}

\author{
Readmission to an Adolescent Psychiatry Inpatient Unit: \\ Readmission Rates and Risk Factors
}

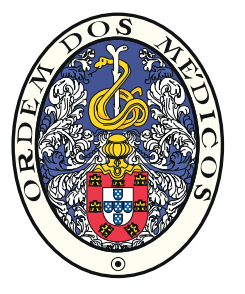

\author{
Patrícia MENDES $\otimes^{1}$, Maria FONSECA ${ }^{1}$, Inês AGUIAR ${ }^{1}$, Nuno PANGAIO ${ }^{1}$, Manuela ARAÚJO ${ }^{1}$, Luísa CONFRARIA ${ }^{1}$, \\ Otília QUEIRÓS ${ }^{1}$, Joana SARAIVA', Pedro MONTEIRO' ${ }^{1}$, João GUERRA ${ }^{1}$ \\ Acta Med Port 2017 Nov;30(11):769-774 - https://doi.org/10.20344/amp.8842
}

\section{RESUMO}

Introdução: A maioria das perturbações mentais tem uma evolução crónica pelo que certos reinternamentos são inevitáveis. Vários estudos indicam taxas de reinternamento pedopsiquiátrico superiores a $25 \%$. O nosso objetivo é calcular as taxas de readmissão no internamento pedopsiquiátrico do Centro Hospitalar do Porto a 30 dias e um ano após a alta, e identificar os fatores de risco associados.

Material e Métodos: A metodologia consistiu na consulta dos processos clínicos dos doentes internados na Unidade de Internamento do Centro Hospitalar do Porto entre 2010 e 2013, a fim de calcular as taxas de readmissão. Foram também recolhidas as características demográficas e clínicas dos doentes readmitidos. Finalmente, os resultados do grupo de doentes reinternados foram comparados com um segundo grupo de doentes selecionados aleatoriamente e sem readmissões hospitalares, a fim de investigar possíveis fatores de risco para reinternamentos.

Resultados: Um total de 445 doentes foi admitido entre 2010 e 2013. Seis adolescentes foram readmitidos no período de 30 dias $(1,3 \%)$ e 52 foram readmitidos nos 12 meses após a alta (11,5\%). A análise comparativa revelou que a duração do internamento e o número de internamentos anteriores são preditores significativos $(p=0,04$ e $p=0,014)$ para reinternamento.

Discussão: As baixas taxas de readmissão podem refletir não só a eficácia da intervenção terapêutica durante o internamento como também um bom suporte clínico e sociofamiliar após a alta.

Conclusão: Os reinternamentos são considerados um alvo fundamental quanto à prevenção e intervenção nos cuidados de saúde mental. Assim, o conhecimento sobre a sua minimização é crucial.

Palavras-chave: Adolescente; Factores de Risco; Hospitalização; Perturbações Mentais; Portugal; Readmissão do Doente; Serviço de Psiquatria

\section{ABSTRACT}

Introduction: Most mental disorders have a chronic evolution and therefore a certain amount of psychiatric readmissions are inevitable. Several studies indicate that over $25 \%$ of child and adolescent inpatients were readmitted within one year of discharge. Several risk factors for psychiatric readmissions have been reported in the literature, but the history of repeated readmissions is the most consistent risk factor. Our aim is to calculate the readmission rates at 30 days and 12 months after discharge and to identify associated risk factors. Material and Methods: The authors consulted the clinical files of patients admitted to the Inpatient Unit between 2010 and 2013 , in order to calculate the readmission rates at 30 days and at 12 months. The demographic and clinical characteristics of the readmitted patients were analyzed and compared with a second group of patients with no hospital readmissions, in order to investigate possible predictors of readmission.

Results: A total of 445 patients were admitted to our inpatient unit between 2010 and 2013. Six adolescents were readmitted in a 30 days period $(1.3 \%)$ and 52 were readmitted in a 12 month period after discharge $(11.5 \%)$. Duration of the hospitalization and the previous number of mental health admissions were significant predictors of future hospital readmissions $(p=0.04$ e $p=0.014)$.

Discussion: The low readmission rates may reflect the positive clinical and sociofamilial support being provided after discharge.

Conclusion: Rehospitalisation is considered a fundamental target for intervention concerning prevention and intervention in mental healthcare. Thus, knowledge regarding their minimisation is crucial.

Keywords: Adolescent; Hospitalization; Mental Disorders; Patient Readmission; Portugal; Psychiatric Department, Hospital; Risk Factors

\section{INTRODUÇÃO}

A maioria das perturbações mentais tem uma evolução crónica, marcada por várias recaídas, pelo que alguns reinternamentos psiquiátricos são inevitáveis. ${ }^{1}$ Vários estudos indicam que mais de $25 \%$ das crianças e adolescentes internados foram readmitidos dentro de um ano após a alta. ${ }^{1,2}$ Estudos mais recentes referem taxas entre $30 \%$ a $50 \%$. $^{3-5}$ Já as taxas de readmissão a 30 dias foram descritas entre $10 \%$ a $14 \% .6,7$

Vários fatores de risco para reinternamento psiquiátrico em adultos têm sido relatados na literatura com resul- tados controversos, tais como: diagnóstico de perturbação psicótica ou perturbação da personalidade, baixa adesão ao tratamento, abuso de drogas, ausência de ocupação, pouco apoio familiar e antecedentes familiares de doença psiquiátrica. ${ }^{3,5,7-9}$ Existem menos estudos sobre os fatores de risco de reinternamento em crianças e adolescentes, mas são apontados os seguintes: gravidade da patologia,, 8 comportamento disruptivo ou graves problemas de conduta, ${ }^{2,5}$ disciplina parental severa, ${ }^{2}$ baixo envolvimento parental, ${ }^{2}$ diagnóstico de perturbação do humor, ${ }^{10}$ perturbação

1. Serviço de Psiquiatria da Infância e da Adolescência. Centro Hospitalar do Porto. Porto. Portugal.

$\square$ Autor correspondente: Patrícia Mendes. titasmendes86@hotmail.com

Recebido: 13 de fevereiro de 2017 - Aceite: 24 de julho de 2017 | Copyright $\odot$ Ordem dos Médicos 2017 
psicótica ou de perturbação do comportamento alimentar (PCA $),{ }^{5}$ institucionalização, ${ }^{5,7}$ comportamentos suicidários, ${ }^{5,7}$ dificuldades de aprendizagem, ${ }^{5}$ duração do internamento $0^{8,9,11} \mathrm{e}$ falta de cuidados pedopsiquiátricos adequados pós-alta. ${ }^{3,5,8,10} \mathrm{~A}$ história de reinternamentos repetidos é o fator de risco mais consistente na literatura. ${ }^{3,5-8}$

Um estudo realizado anteriormente nesta unidade de internamento (UI), então integrada no Hospital Central Especializado de Crianças Maria $\mathrm{Pia}$, com a população de doentes internados entre 1999 e 2003 identificou os seguintes fatores de risco para reinternamento: diagnóstico de perturbação da personalidade, PCA ou perturbação psicótica e duração do internamento prévio superior a 30 dias (Teles A, Martins V, Guerra J, Fontes C, Rodrigues AC. Comunicação pessoal e apresentação de poster no XV Encontro Nacional de Psiquiatria da Infância e da Adolescência).

Não foram encontrados na literatura outros trabalhos acerca das readmissões hospitalares pedopsiquiátricas em Portugal. Uma das dificuldades na generalização dos estudos internacionais prende-se com as especificidades de cada unidade de internamento, visto a maioria ser especializada em determinada patologia ou faixa etária dos doentes admitidos. A Unidade de Internamento (UI) do Departamento de Psiquiatria da Infância e da Adolescência do Centro Hospitalar do Porto foi criada em 1974 e atualmente tem a capacidade de 10 camas. Esta unidade presta cuidados médicos psiquiátricos a jovens com idades compreendidas entre os 12 e os 17 anos inclusive, residentes na zona norte embora possam ser admitidos jovens de outros locais do país. Excecionalmente podem ser admitidas crianças mais novas se clinicamente justificado. A equipa técnica é constituída por uma equipa multidisciplinar, constituída por médicos pedopsiquiatras, psicólogas clínicas, assistente social, animadora sociocultural e uma equipa de enfermagem. A unidade disponibiliza abordagens psicoterapêuticas específicas para os doentes e para as suas famílias, nomeadamente abordagens individuais e familiares, grupo de expressão plástica, grupo terapêutico de doentes internados e grupo de pais de jovens internados. Sempre que necessário, é solicitada intervenção terapêutica em Terapia Familiar e Psicodrama Moreniano existentes no departamento.

A intervenção em internamento tem critérios restritos e ocorre tipicamente quando outras formas de tratamento são insuficientes, quando está em causa a segurança do jovem ou em casos de descompensação psicopatológica grave. $\mathrm{O}$ facto de constituir um espaço protegido, contentor e estruturado configura a UI ainda como meio preferencial para esclarecimento de diagnósticos e instituição de tratamentos intensivos, permitindo rápidos ajustes da terapêutica farmacológica. É de referir que a unidade não se encontra vocacionada para o tratamento de toxicodependências, nem para situações de acolhimento residencial de jovens em situação social de risco ou que necessitem de implementação de medidas educativas. A UI permite, assim, avaliar jovens com sintomas psiquiátricos graves, clarificar quadros diagnósticos complexos e estabelecer um tratamento adequado num ambiente protegido e especializado.

O nosso objetivo é calcular as taxas de readmissão desta unidade de internamento generalista em dois momentos, a 30 dias e um ano após a alta e identificar fatores de risco associados.

\section{MATERIAL E MÉTODOS}

De forma a atingir os objetivos propostos, realizamos a consulta dos processos clínicos dos doentes internados na UI entre janeiro de 2010 e dezembro de 2013, num período de follow-up de 12 meses após a alta, a fim de calcular as taxas de readmissão em 30 dias e aos 12 meses. Nos casos de jovens que tiveram alta para um serviço de psiquiatria de adultos no período de follow-up de 12 meses, os dados relativos a um possível reinternamento foram consultados na Plataforma de Dados da Saúde (PDS).

Foram consultadas as características sociodemográficas e clínicas dos doentes readmitidos no período de um ano após a alta, constituindo o 'Grupo Reinternamentos' (n =51). Foram obtidos dados relativos ao género, idade, ocupação, cuidador principal, distrito de origem, origem e motivo de internamento, duração do internamento, número de internamentos psiquiátricos anteriores, presença de abuso de substâncias, história de tentativas de suicídio, história familiar de doença mental e cuidados pós-alta do Internamento Index (II). O II corresponde à primeira admissão do paciente durante o período de estudo (janeiro de 2010 a dezembro de 2013), seguido ou não por uma readmissão no prazo de um ano, independentemente de corresponder a um primeiro internamento pedopsiquiátrico ou a um reinternamento. Consideramos apenas o diagnóstico principal, isto é, o diagnóstico responsável pelos sintomas que motivaram o internamento e posteriormente categorizámos segundo os critérios da DSM IV-TR de forma a permitir o tratamento estatístico. A DSM IV-TR era a classificação mais atual publicada à data do período do estudo e em utilização na Unidade. Não foram consideradas as comorbilidades diagnósticas.

Finalmente, comparámos os resultados do primeiro grupo de doentes com, pelo menos, uma readmissão hospitalar dentro de um ano após a alta do internamento com um segundo grupo de doentes, selecionados aleatoriamente do total de doentes sem readmissões nesse período de tempo, denominado 'Grupo Sem Reinternamentos' ( $n=$ 58). O tamanho da amostra sem reinternamentos foi selecionado de forma a corresponder aproximadamente ao tamanho do grupo de casos reinternados.

A análise estatística foi realizada com recurso ao SPSS 20.0. O teste $t$-student foi realizado para comparar variáveis contínuas e o teste qui-quadrado para avaliar a associação entre variáveis categóricas. Realizou-se a regressão logística utilizando a duração do internamento e o número de internamentos anteriores. A significância estatística foi considerada quando $p<0,05$. 


\section{RESULTADOS}

Foram admitidos 445 doentes no período do estudo (2010 - 2013). Verificaram-se apenas seis readmissões hospitalares nos 30 dias após a data da alta, correspondendo a uma taxa de reinternamento de 1,3\%. No período de um ano após a alta hospitalar foram readmitidos 51 doentes, pelo que se verificou uma taxa de reinternamento a um ano de $11,5 \%$.

Os seis reinternamentos nos 30 dias pós-alta foram verificados entre o $11^{\circ}$ e $24^{\circ}$ dia e correspondiam aos seguintes diagnósticos: perturbação invasiva do desenvolvimento (dois casos), perturbação do humor (dois casos), perturbação psicótica (um caso) e perturbação de ansiedade (um caso).

Apenas 17 reinternamentos ocorreram nos primeiros três meses após a alta, isto é, apenas 1/3 foram reinternamentos precoces (normalmente definidos como $<90$ dias após a alta).

Na Fig. 1 encontra-se a distribuição dos doentes reinternados por categorias diagnósticas. As categorias mais prevalentes neste grupo são as PCA e as perturbações psicóticas.

É de realçar que os diagnósticos que motivaram o internamento podem corresponder a descompensações face a stressores externos de doentes com outra patologia base, potencialmente até de maior cronicidade e gravidade, nesse momento compensada. Por se encontrar fora do âmbito deste trabalho não se investigou se o diagnóstico no reinternamento correspondia ao mesmo diagnóstico no II ou a outro quadro clínico. Por exemplo, os diagnósticos de perturbação de adaptação correspondiam, na sua maioria a jovens com comorbilidades importantes, mas que não refletiam, no II, o motivo de internamento. Assim, os doentes apresentavam como comorbilidades na data do II os

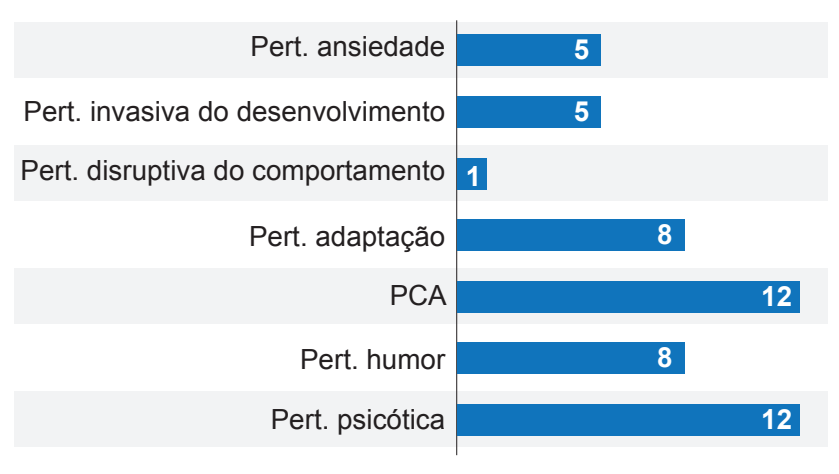

Figura 1 - Categorias diagnósticas, segundo a DSM IV-TR, dos doentes reinternados no período de um ano após alta do internamento pedopsiquiátrico

diagnósticos de perturbação da personalidade estado limite (um caso), perturbação psicótica sem outra especificação (dois casos), deficiência mental (um caso) e perturbação de ansiedade (dois casos). É de realçar que o diagnóstico de perturbação de adaptação pode ser considerado em jovens com traços disfuncionais da personalidade mas que ainda não preenchiam critérios para este diagnóstico na data do II. Efetivamente em dois outros casos foi efetuado o diagnóstico de perturbação da personalidade apenas aquando da readmissão e considerando a evolução do quadro após oll.

Já os casos considerados na categoria das perturbações de ansiedade diziam respeito a três diagnósticos de perturbação obsessivo-compulsiva, um diagnóstico de estado de ansiedade não especificado (neste caso com comorbilidade de perturbação de personalidade do tipo evitante) e um caso de estado fóbico não especificado.

Na Tabela 1 encontram-se os dados sociodemográficos de ambos os grupos de doentes.

Tabela 1 - Dados clínicos e sociodemográficos de ambos os grupos de doentes

\begin{tabular}{|c|c|c|c|c|c|}
\hline \multirow[b]{2}{*}{ Género } & \multirow[b]{2}{*}{ Masculino } & \multicolumn{2}{|c|}{$\begin{array}{l}\text { Grupo Reinternamentos } \\
\qquad(\mathrm{n}=51)\end{array}$} & \multicolumn{2}{|c|}{$\begin{array}{l}\text { Grupo sem Reinternamentos } \\
\qquad(n=58)\end{array}$} \\
\hline & & 16 & $(31,4 \%)$ & 23 & $(39,7 \%)$ \\
\hline & Feminino & 35 & $(68,6 \%)$ & 35 & $(60,3 \%)$ \\
\hline \multirow[t]{2}{*}{ Idade } & $<15$ anos & 18 & $(35,3 \%)$ & 14 & $(24,1 \%)$ \\
\hline & $15-18$ anos & 33 & $(64,7 \%)$ & 44 & $(75,9 \%)$ \\
\hline \multirow[t]{2}{*}{ Cuidador } & País & 49 & $(96,1 \%)$ & 51 & $(87,9 \%)$ \\
\hline & Outros familiares/Instituição & 2 & $(4,0 \%)$ & 7 & $(12,0 \%)$ \\
\hline Abuso de substâncias & & 4 & $(7,8 \%)$ & 10 & $(17,2 \%)$ \\
\hline Fraca adesão terapêutica & & 20 & $(39,2 \%)$ & 13 & $(22,4 \%)$ \\
\hline Risco social & & 20 & $(39,2 \%)$ & 24 & $(41,4 \%)$ \\
\hline Ideação suicida & & 16 & $(31,4 \%)$ & 23 & $(39,7 \%)$ \\
\hline Comportamento agressivo & & 13 & $(25,5 \%)$ & 14 & $(24,1 \%)$ \\
\hline Admissões prévias* & & 14 & $(26,5 \%)$ & 2 & $(3,4 \%)$ \\
\hline História familiar psiquiátrica & & 31 & $(60,8 \%)$ & 30 & $(51,7 \%)$ \\
\hline Dificuldades de aprendizagem* & & 23 & $(45,1 \%)$ & 15 & $(25,9 \%)$ \\
\hline $\begin{array}{l}\text { Duração do internamento } \\
\text { (média } \pm d p)^{*}\end{array}$ & & & $=36,29$ & & 25,89 \\
\hline
\end{tabular}

*Diferenças estatisticamente significativas 
Não foram encontradas diferenças estatisticamente significativas entre os grupos em relação aos cuidadores, idade, ocupação, origem da admissão, comportamento agressivo, abuso de substâncias, adesão terapêutica, risco social, ideação suicida, número de comorbilidades, presença de antecedentes familiares psiquiátricos, acompanhamento anterior em serviços de saúde mental, prescrição de medicação depôt, orientação pós-alta ou tempo decorrido até a reavaliação pós-alta.

Foram encontradas diferenças estatisticamente significativas entre os grupos relativamente a:

- Internamentos prévios ao II. O Grupo Readmitidos teve mais admissões hospitalares prévias $(p<0,05)$;

- Duração do internamento no II $(p<0,05)$. Os doentes readmitidos apresentaram maiores tempos de internamento;

- Categoria diagnóstica $(p=0,056)$. As PCA e as perturbações psicóticas parecem ser mais frequentes nos doentes readmitidos;

- Dificuldades de aprendizagem $(p<0,05)$. Os doentes reinternados apresentavam mais retenções e necessidades educativas especiais.

Usando uma regressão logística foi avaliado se o reinternamento poderia ser previsto pela duração do internamento e pelo número de internamentos prévios. Todos os requisitos foram cumpridos. $\mathrm{O}$ modelo foi estatisticamente significativo $\chi^{2}=16,65, p<0,05$ e classifica corretamente $67,9 \%$ dos casos.

A duração do internamento e o número de internamentos prévios foram preditores significativos $(p=0,04$ e $p=$ 0,014 , respetivamente). Com o aumento da duração do internamento, aumenta também o risco de reinternamento (OR: 1,02). Com o aumento do número de internamentos prévios, aumenta também o risco de um novo reinternamento (OR: 4,99).

\section{DISCUSSÃO}

As taxas de reinternamento obtidas na nossa Unidade de Internamento são marcadamente inferiores às descritas na literatura, $1,3 \%$ vs $10 \%-14 \%$ a 30 dias e $11,5 \%$ vs $25 \%-50 \%$ a um ano. ${ }^{1-7}$ Os autores apontam como possíveis explicações o bom e rápido suporte clínico e sociofamiliar após a alta hospitalar, os esforços para reavaliações pedopsiquiátricas frequentes e o importante trabalho de coordenação com instituições e serviços da comunidade, promovendo a inserção social e o apoio às famílias e permitindo a manutenção dos cuidados em ambulatório, prevenindo assim reinternamentos frequentes.

Os resultados divergem da literatura que aponta que o risco de reinternamento em idade pediátrica é mais provável no primeiro trimestre após a alta. ${ }^{2,5} \mathrm{~A}$ boa articulação entre a unidade de atendimento urgente pedopsiquiátrica que é responsável pela maioria das admissões hospitalares e a equipa pedopsiquiátrica responsável pelo acompanhamento dos doentes adolescentes, com possibilidade de comunicação fácil com o pedopsiquiatra assistente, antecipação de consulta ou agendamento de consulta de crise num curto período de tempo pode também prevenir algumas readmissões hospitalares.

Para além dos fatores já apontados, é nossa convicção que certos fatores inerentes à equipa terapêutica e à abordagem de certas patologias podem contribuir para as baixas taxas de reinternamento, nomeadamente, o estabelecimento de critérios de internamento apurados e eficazes, a agilização do tratamento e da resposta após a alta. Quanto aos comportamentos autolesivos com ou sem ideação suicida e traços disfuncionais de personalidade na adolescência, a experiência na UI levou à convicção da ineficácia e/ou prejuízo da admissão hospitalar em muitos dos casos. Nos casos de admissão procura-se que o internamento seja curto e com objetivos claros e bem definidos de forma a evitar potenciais consequências negativas do mesmo (desintegração sócio-escolar, desresponsabilização das tarefas quotidianas e escolares, fuga a stressores existente no contexto do jovem, distanciamento da família com risco de enquistamento das dificuldades relacionais, etc.). Trata-se de uma população especialmente propensa aos fenómenos 'porta giratória' (doentes ciclicamente readmitidos) dada a ocorrência de crises recorrentes e o internamento pode, paradoxalmente, reforçar mecanismos de resolução de problemas mal-adaptivos (evitamento, reforço do estatuto de doente...). Daí ser fundamental o desenvolvimento e implementação de intervenções em ambulatório para estes doentes.

A experiência da equipa da UI, assim como a abordagem e intervenção adotadas, são suportadas pelas diversas guidelines de intervenção nas perturbações da personalidade na adolescência (NICE, IACAPAP) que defendem que o tratamento deve ser prestado preferencialmente em ambulatório. Segundo estas, a admissão hospitalar só deve ser considerada no caso de comorbilidade severa ou quando em situação de crise com elevado risco para o paciente em que a contenção não seja possível em ambulatório. ${ }^{12,13}$

A maioria dos fatores de risco identificados na literatura são inconsistentes. Os nossos resultados mostram uma tendência estatística para as PCA e as dificuldades de aprendizagem representarem fatores de risco, mas são necessários mais estudos para estabelecer esta relação de forma consistente.

O número de internamentos prévios é o fator de risco de readmissão mais consistentemente considerado na literatura. ${ }^{3,5}$ Os resultados deste trabalho suportam esta associação em idade pediátrica. Os reinternamentos psiquiátricos frequentes correlacionam-se com resultados desfavoráveis. ${ }^{2}$ Portanto, adolescentes com mais do que um internamento devem ser rápida e vigorosamente avaliados e orientados a fim de evitar o fenómeno 'porta giratória' nas unidades de internamento.

Quanto à duração do internamento, a literatura é pouco consistente. . $, 8,11$ Alguns estudos apontam como fator de risco o internamento prolongado, embora apontem como viés a maior gravidade da patologia. ${ }^{14-18}$ Por outro lado, internamentos de curta duração também foram associados a um maior risco de internamento pelo risco de não se ter 
atingido a estabilização da doença aquando da alta..$^{9,19}$

De acordo com os nossos resultados, a duração de internamento prolongada aumenta o risco de uma futura readmissão. Note-se que as hospitalizações longas foram associadas principalmente aos diagnósticos de PCA e Perturbação Psicótica, logo, a maior duração do internamento pode efetivamente refletir uma maior gravidade da doença. São necessários estudos mais detalhados de forma a controlar estes possíveis fatores de confundimento. Já as hospitalizações curtas não foram associadas a um risco aumentado de reinternamento. Estes internamentos de menor duração correspondem frequentemente a casos de admissão para esclarecimento diagnóstico e orientação terapêutica a ser implementada em ambulatório sempre que possível.

O estudo prévio realizado na UI entre 1999 e 2003 identificou como fatores de risco para reinternamento, para além da duração do internamento prévio superior a 30 dias, os diagnósticos de perturbação da personalidade, PCA ou perturbação psicótica (Teles A, Martins V, Guerra J, Fontes C, Rodrigues AC. Comunicação pessoal e apresentação de poster no XV Encontro Nacional de Psiquiatria da Infância e da Adolescência). Neste estudo, a categoria diagnóstica não demonstrou ser uma variável estatisticamente significativa, embora as PCA apresentem uma tendência estatística nesse sentido. Isto pode dever-se a diferenças na metodologia entre os estudos ou à pequena dimensão da nossa amostra.

Os reinternamentos psiquiátricos refletem a influência conjunta de fatores clínicos, como categoria diagnóstica ou gravidade da doença, e fatores contextuais pelo que é difícil avaliar todos os possíveis elementos de risco ou proteção. Algumas variáveis podem não demonstrar significância estatística devido ao baixo número de doentes admitidos no estudo e certas variáveis não estudadas podem, também, ter interferido com os resultados (por exemplo: stress familiar, capacidade económica, gravidade dos sintomas, capacidade cognitiva...). Este estudo tem como limitações o fato de ter considerado apenas o diagnóstico principal, excluindo a avaliação das comorbilidades psiquiátricas, a seleção aleatória do grupo de doentes não readmitidos e não utilização da população total de doentes não readmitidos para comparação com o grupo de doentes readmitidos.

No futuro, seria ainda de grande interesse a obtenção de dados relativos à natureza dos reinternamentos e a sua comparação com os internamentos prévios.

\section{CONCLUSÃO}

Os resultados sugerem que informações obtidas durante o internamento (diagnóstico, duração da admissão, número de internamentos anteriores) podem fornecer um índice útil na avaliação do risco de reinternamentos posteriores.

Os reinternamentos são considerados um alvo fundamental para análise e prevenção nos cuidados de saúde. Assim, o conhecimento sobre a sua minimização é crucial. Este estudo permite um maior conhecimento das características da população de doentes reinternados na nossa unidade e identifica fatores de risco facilmente detetáveis durante a hospitalização permitindo a sua abordagem e a adoção de medidas preventivas nos casos em maior risco de reinternamento.

\section{PROTECÇÃO DE PESSOAS E ANIMAIS}

Os autores declaram que os procedimentos seguidos estavam de acordo com os regulamentos estabelecidos pelos responsáveis da Comissão de Investigação Clínica e Ética e de acordo com a Declaração de Helsínquia da Associação Médica Mundial.

\section{CONFIDENCIALIDADE DOS DADOS}

Os autores declaram ter seguido os protocolos do seu centro de trabalho acerca da publicação de dados.

\section{CONFLITOS DE INTERESSE}

Os autores declaram não terem qualquer conflito de interesse relativamente ao presente artigo.

\section{FONTES DE FINANCIAMENTO}

Os autores declaram não ter recebido subsídios ou bolsas para a elaboração do artigo.

\section{REFERÊNCIAS}

1. Asarnow JR, Goldstein MJ, Carlson GA, Perdue S, Bates S, Keller J Childhood-onset depressive disorders. A follow-up study of rates of rehospitalization and out-of-home placement among child psychiatric inpatients. J Affect Disord. 1988;15:245-53.

2. Blader JC. Symptom, family, and service predictors of children's psychiatric rehospitalization within one year of discharge. J Am Acad Child Adolesc Psychiatry. 2004;43:440-51.

3. Lien L. Are readmission rates influenced by how psychiatric services are organized? Nord J Psychiatry. 2002;56:23-8.

4. Mgutshini T. Risk factors for psychiatric re-hospitalization: an exploration. Int J Ment Health Nurs. 2010;19:257-67.

5. Tossone K, Jefferis E, Bhatta MP, Bilge-Johnson S, Seifert P. Risk factors for rehospitalization and inpatient care among pediatric psychiatric intake response center patients. Child Adolesc Psychiatry Ment Health. 2014;8:27.

6. Zilber N, Hornik-Lurie T, Lerner Y. Predictors of early psychiatric rehospitalization: a national case register study. Isr J Psychiatry Relat
Sci. 2011;48:49-53.

7. Callaly $\mathrm{T}$, Hyland M, Trauer T, Dodd S, Berk M. Readmission to an acute psychiatric unit within 28 days of discharge: identifying those at risk. Aust Health Rev Publ Aust Hosp Assoc. 2010;34:282-5.

8. Yampolskaya S, Mowery D, Dollard N. Predictors for readmission into children's inpatient mental health treatment. Community Ment Health J. 2013;49:781-6.

9. Figueroa R, Harman J, Engberg J. Use of claims data to examine the impact of length of inpatient psychiatric stay on readmission rate. Psychiatr Serv. 2004;55:560-5.

10. Foster EM. Do aftercare services reduce inpatient psychiatric readmissions? Health Serv Res. 1999;34:715-36.

11. Wickizer TM, Lessler D, Boyd-Wickizer J. Effects of health care costcontainment programs on patterns of care and readmissions among children and adolescents. Am J Public Health. 1999;89:1353-8.

12. National Institute for Health and Care Excellence: Borderline personality disorder: treatment and management. NICE clinical guideline 782009. 
[consultado 2016 nov 26]. Disponível em: https://www.nice.org.uk/ guidance/cg78.

13. Cailhol L, Gicquel L, Raynaud JP. Borderline personality disorder IACAPAP e-Textbook of Child and Adolescent Mental Health. Rey JM, editor. Geneva: International Association for Child and Adolescent Psychiatry and Allied Professions; 2015. [consultado 2016 nov 26]. Disponível em: http://iacapap.org/wp-content/uploads/H.4BORDERLINE-PD-072012.pdf.

14. Thompson EE, Neighbors HW, Munday C, Trierweiler S. Length of stay, referral aftercare, and rehospitalization among psychiatric inpatients. Psychiatr Serv. 2003;54:1271-6.

15. Hodgson RE, Lewis M, Boardman AP. Prediction of readmission to acute psychiatric units. Soc Psychiatry Psychiatr Epidemiol. 2001;36:304-9.
16. Korkeila JA, Lehtinen V, Tuori T, Helenius H. Frequently hospitalized 10 psychiatric patients: a study of predictive factors. Soc Psychiatry Psychiatr Epidemiol. 1998;33:528-34.

17. Mojtabai R, Nicholson RA, Neesmith DH. Factors affecting relapse in patients discharged from a public hospital: results from survival analysis. Psychiatr Q. 1997;68:117-29.

18. Qiesvold T, Saarento O, Sytema S, Vinding H, Göstas G, Lönnerberg $O$, et al. Predictors for readmission risk of new patients: The Nordic comparative study on sectorized psychiatry. Acta Psychiat Scand. 2000;101:367-73.

19. Lin HC, Tian WH, Chen CS, Liu TC, Tsai SY, Lee HC. The association between readmission rates and length of stay for schizophrenia: A 3-year population-based study. Schizophr Res. 2006;83:211-4. 